\title{
El uso del Tangram como estrategia de aprendizaje para el desarrollo de la creatividad y las inteligencias múltiples
}

Claudia Janneth Piraquive Peña, Verónica López Fernández y Fátima Llamas Salguero, Universidad Internacional de la Rioja (UNIR), España

Recepción: 12 de mayo de 2015 | Revisión: 17 de mayo de 2015 | Aceptación/Publicación: 6 de junio de 2015

Correspondencia: veronica.lopez@unir.net

Citar: Piraquive Peña, C.J., López Fernández, V. y Llamas Salguero, F. (2015). El uso del Tangram como estrategia de aprendizaje para

el desarrollo de la creatividad y las inteligencias múltiples. ReiDoCrea, 4, 74-84.

\section{Resumen}

Este trabajo pretende comprobar si la aplicación de un programa de intervención basado en la utilización del Tangram, potencia y desarrolla las inteligencias múltiples, la creatividad y la motivación de los alumnos. Para ello, se ha seguido un diseño pre y post-test en el que se ha seleccionado una muestra de 40 estudiantes de grado sexto de la Institución Educativa General Santander del municipio de Soacha, Colombia, a quienes se les aplica una serie de pruebas para evaluar las variables mencionadas. Además se evalúa la percepción viso-motriz para comprobar si hay correlación entre ella, las inteligencias, la motivación y la creatividad. Los resultados confirman la existencia de una relación estadísticamente significativa únicamente entre la creatividad y la motivación, además se evidencia la mejora tanto en las inteligencias múltiples como en la creatividad, pero no en la motivación tras la intervención con el Tangram. Concluyendo la enseñanza lúdica puede ser un recurso de ayuda para potenciar y desarrollar las inteligencias múltiples en los estudiantes, así como su creatividad.

Palabras clave: Motivación | Creatividad | Inteligencias | Tangram

\section{Abstract}

This work aims to verify whether the application of an intervention program based on the use of the Tangram, power and develops multiple intelligences, creativity and motivation of students. To do so, it has followed a pre posttest design in which we have selected a sample of 40 sixth grade students of School General Santander in the municipality of Soacha, Colombia, to whom it is applied a series of tests for assess these variables. Besides visual-motor perception to check correlation between it, the intelligence, motivation and creativity is evaluated. The results confirm the existence of a statistically significant relationship only between creativity and motivation; besides the improvement it is evident both in the multiple intelligences and creativity, but not the motivation behind the intervention with Tangram. Concluding the edutainment can be a source of help to enhance and develop multiple intelligences in students as well as their creativity.

Keywords: Motivation | Creativity | Intelligences | Tangram

\section{Introducción}

El ser humano se ha caracterizado por expresar sus habilidades y sobresalir en ellas, pero el sistema educativo no ha contribuido a este fin, pues no siempre se atienden todas las capacidades de todos los estudiantes, de hecho, en la mayoría de los casos, la dinámica de trabajo aborda el aprendizaje como si todos los estudiantes tuvieran las mismas capacidades y necesitaran adquirir las mismas herramientas para enfrentarse al mundo. El hecho de no contemplar estas diferencias puede ocasionar en los estudiantes 
falta de interés por aprender y por dominar sus habilidades. Tal vez se deba al enfoque de la educación en Colombia, ya que éste se ha centrado en considerar al estudiante como receptor, al docente como emisor y el conocimiento como una camisa de fuerza, es decir, se observa, se escribe y se repite (Pradas y Pardo, 2013) Una evidencia de lo anterior son los bajos resultados de las pruebas Saber y la prueba PISA, especialmente en el área de matemáticas en los estudiantes de Colombia y otros países. Por supuesto, existen otros factores que influyen como son el nivel económico del hogar, nivel educativo de los padres, las proyecciones de cada alumno, los medios de comunicación, las políticas educativas, los objetivos de la escuela, el contexto o el medio donde se encuentran, las concepciones de los docentes, etc. Pero gracias a las investigaciones de Gardner (1995), se incluyó que los seres humanos tenemos ocho inteligencias, las cuales se desarrollan de acuerdo a las oportunidades de aprendizaje que se tengan, no todos aprenden lo mismo, al mismo ritmo y de la misma forma. A partir de lo anterior se debe replantear la metodología del aula. El primer paso es la motivación, el estudiante necesita un ambiente seguro para formarse con la presencia de las competencias emocionales (García y Doménech, 2012). El segundo paso es tener presente no solo la necesidad de trasmitir conocimientos, sino que debemos tener en cuenta el desarrollo creativo del estudiante y un último paso, es crear situaciones en las cuales el estudiante tenga la oportunidad de desarrollar sus inteligencias.

La enseñanza de las Matemáticas en Colombia ha evolucionado en las últimas décadas en cuanto al diseño curricular. Las instituciones educativas tienen autonomía para crear su proyecto educativo institucional acorde a los lineamientos y estándares del MEN (Ministerio de Educación Nacional), lo ideal es que los docentes sean quienes lo realicen, para que su quehacer en el aula evidencie las innovaciones en el proceso de enseñanza y aprendizaje, evitando la monotonía, el desinterés y por el contrario fortaleciendo el pensamiento matemático (Jaramillo y Ruíz, 2012). No obstante, esto no siempre ocurre y se continua con una metodología tradicional "memorística" alejada de la realidad, lo que genera desinterés y bajo rendimiento, esto se observa en el poco ingreso a la educación superior y en los resultados de la prueba Saber y PISA (Linares, 2013). Precisamente, porque el interés del alumnado ha de tenerse en cuenta, en el siguiente apartado se explica la importancia de este factor en el aula.

La motivación es una posible explicación de la conducta humana en diferentes situaciones. Esta puede ser consciente como los gustos, intereses y propósitos, en este caso muestra una relación directa entre lo que se desea y lo que se realiza, es decir, la motivación hace que un sujeto inicie una acción utilizando sus competencias la direccione hacia un objetivo y se mantenga hasta lograrlo (McClelland, 1989). Enfocando al ámbito escolar, la motivación del estudiante en gran parte depende de sus actitudes para alcanzar sus metas y la otra parte obedece a la influencia del entorno y su interacción con otros para obtener una recompensa. Aquí juega un papel importante las emociones en el rendimiento escolar, las positivas, como la esperanza antes de realizar la tarea para conseguir el éxito y disfrute al realizarla; mientras, las negativas como el aburrimiento y la ansiedad por evitar el fracaso, puede beneficiar pero en un momento, pueden llegar a bloquear y por el contrario impiden realizar la tarea (Pekrun, 1992). Esta idea es utilizada para explicar el alto y bajo rendimiento en los estudiantes, donde el éxito lo justifican por el esfuerzo y la alta capacidad, mientras el bajo por la mala suerte e incapacidad (Weiner, 1974).

Respecto a la creatividad, una de las teorías clásicas más aceptada es publicada por Graham Wallas en 1926, esta consta de cuatro etapas secuenciales y aunque existen 
otras investigadores desde diferentes enfoques, se concluye la creatividad es la capacidad para resolver problemas de manera novedosa, que toda la humanidad la posee en mayor o menor grado, dependiendo de las inteligencias que tenga desarrolladas (Gardner,1999).Teniendo en cuenta lo anterior, los docentes deben desarrollar la capacidad creativa del estudiante, en un ambiente agradable y seguro, iniciando con una consulta previa del tema, proponiendo actividades lúdicas con un tiempo prudente para reflexionar y comprender el problema, seguido de un espacio para estimular la curiosidad, planteando interrogantes, proponiendo posibles soluciones y comprobándolas, para seleccionar la más adecuada. Si se practica lo anterior se estará desarrollando la creatividad, es decir, un pensamiento crítico, flexible y original (Ortega, 2012).

Es fundamental, por lo tanto, incentivar la creatividad en el aula. Este proceso cognitivo complejo, cuenta con la participación de varias estructuras cerebrales. Así, la creatividad es una interacción entre amígdala, hipocampo, córtex prefrontal y neuronas con el objetivo de plantear hipótesis e ideas originales. De esta manera, las investigaciones afirman que los sujetos creativos presentan alta actividad cerebral conscientes o inconscientes y esta se incrementa cuando se enfrenta a solucionar un problema (Montilla, 1997).

En relación, con inteligencias múltiples Gardner (1983), afirma que un sujeto normal tiene ocho inteligencias, pero unas más desarrolladas que las otras, resaltando la influencia que tiene la herencia, la educación y el ambiente para el desarrollo de las mismas, aclara que están distribuidas en diferentes regiones cerebrales funcionando de manera independiente y combinada. También afirma, un sujeto es único con distintas capacidades para tomar decisiones, solucionar situaciones o crear un producto útil a la comunidad. Por tanto es fundamental promover en los estudiantes actividades que desarrollen todas las inteligencias.

Este estudio también ha querido incluir la variable percepción viso-motora, que es la habilidad de coordinar movimientos simultáneos de ojo y mano con precisión en un espacio determinado, al igual, que crear en su mente la acción antes de efectuarse. Para que las actividades se desarrollen, es necesario tener visión adecuada, regulación del sistema nervioso central, coordinación motriz de tronco, brazo, mano y dedos Esta coordinación evoluciona con la edad y con el entrenamiento, aunque depende de factores externos como la motivación, el grado de complejidad y de factores internos como el grado de maduración neurológica y nivel cognitivo (Cruz, Garaigordobil y Maganto, 2001). En la práctica educativa, se observa en algunas ocasiones como los estudiantes que presentan dificultades viso-motora se desaniman porque se les corrige constantemente y se les dificulta el desarrollo de actividades, y ello puede facilitar bajo rendimiento académico.

En relación al uso del Tangram, los investigadores Cabrera Astudillo, Lluilema y Carmen (2011) evidencian que los estudiantes que manipulan material concreto tienen disposición por aprender. Otro estudio realizado por Jaramillo (2013) muestran la eficacia de una metodología participativa usando el tangram, en cuanto a que ayuda al docente a comunicarse eficazmente con los estudiantes y éstos a la vez serán capaces de asimilar el conocimiento necesario y reflejarlo tanto en su rendimiento así como en su conducta, fomentando un mejor aprendizaje. 
Es así como esta investigación pretende brindar una herramienta como estrategia de aprendizaje al docente para que motive al estudiante a través de la manipulación de material concreto específicamente el Tangram, y a su vez se muestren las matemáticas de manera interesante, real y hasta divertida, utilizando diferentes estrategias que faciliten el aprendizaje, donde el estudiante sea un protagonista inquieto e interesado por su desarrollo. Por ello, se pretende la aplicación de un programa de intervención en una muestra y con el análisis estadístico de sus resultados pretest y postest demostrar la efectividad de este recurso didáctico, es decir, en la creatividad y el desarrollo de las inteligencias múltiples.

\section{Método}

\section{Participantes}

La muestra estuvo conformada por 40 estudiantes grado $6^{\circ}$ ( 1 ESO), 18 niñas y 22 niños con edades entre los 11 y 12 años. Pertenecientes a la Institución Educativa General Santander jornada mañana, ubicado en el municipio de Soacha en Colombia, de familias de estrato socioeconómico de uno a tres.

\section{Instrumentos de medida}

Para llevar a cabo este estudio se han empleado los siguientes instrumentos de evaluación:

- Escala Atribucional de Motivación de Logro (EAML), de Manassero y Vázquez (1997, 1998). Consta de 22 ítems, con valoración de 1 a 9 puntos, en orden ascendente o descendente evitando sesgos, estos ítems distribuidos en cinco subescalas, también tiene dos ítems de selección múltiple con única respuesta relacionada con la valoración bimestral de la institución.

- Cuestionario de Creatividad de Turtle (Turtle, 1980). Este es utilizado a partir de quinto de primaria. Consta de 31 ítems con respuesta dicotómica (sí/no), se puede afirmar que tener 12 o más de las características podría indicar un alto nivel de creatividad.

- Cuestionario de detección de las inteligencias múltiples para estudiantes de secundaria, versión adaptada de McKenzie (1999). Mide el desarrollo de las inteligencias múltiples. Se divide en ocho subcuestionarios, cada uno tiene diez ítems enfocadas a cada inteligencia el resultado final de cada inteligencia será la sumatoria de las respuestas afirmativas.

- Test de Bender o Test Guestáltico (Bender, 1977). Tiene como objetivo medir la maduración perceptiva visomotriz en los niños. Parte del supuesto de que cualquier comportamiento responde a un comportamiento total integrado. Este test se aplica individualmente, consta de nueve láminas cada una con un dibujo abstracto. El resultado del test es la sumatoria de puntos, cada punto muestra las dificultades en la copia del dibujo.

\section{Diseño}

Se trata de un diseño cuasi-experimental con un grupo natural empleando metodología pre y post-test y un análisis descriptivo y correlacional. 


\section{Procedimiento}

La primera fase, se explica el objetivo de esta investigación y se obtiene el consentimiento cada participante. Posteriormente, se aplica la prueba de motivación: se dan las instrucciones, se aclaran las dudas y se indica 30 minutos para contestar, se recoge y se les entrega el test de creatividad con tiempo de 20 minutos lo devuelven y se finaliza con el test de inteligencias múltiples con una duración de 20 minutos. En segunda fase, se aplicó el test de Bender, este se administró de manera individual el estudiante se ubicó en un escritorio con hojas blancas, lápiz y borrador. Se le muestra una lámina para que la observe y dibuje, así sucesivamente hasta completar los nueve dibujos abstractos, en promedio se utilizó seis minutos por estudiante. La tercera fase fue el desarrollo de programa de intervención, este consto de seis sesiones dos por semana, cada una de estas sesiones tiene una duración de 45 minutos. En cada sesión se trabaja una actividad específica, algunas individuales y otras en grupo, pero todas centradas en la utilización del tangram. En la cuarta fase se finaliza aplicando nuevamente los test, estos fueron aplicados con la misma metodología que en el pretest.

\section{Resultados}

Para analizar los datos se empleó el programa IBM SPSS Statistics v.20, tanto para el análisis de correlación entre variables como las comparaciones de las variables pre y post-test.

\section{Resultados descriptivos PRETEST}

A continuación se muestran los resultados descriptivos obtenidos en cada una de las variables medidas. En la Tabla 1 puede apreciarse la media, la desviación típica y la moda de cada una de las inteligencias múltiples obtenidas en la fase pretest.

\begin{tabular}{|c|c|c|c|c|c|c|c|c|c|}
\hline \multicolumn{10}{|c|}{$\begin{array}{c}\text { Tabla } 1 \\
\text { Descripción de inteligencias múltiples }\end{array}$} \\
\hline \multirow[b]{2}{*}{ PARAMETROS } & \multirow[b]{2}{*}{ EDAD } & \multicolumn{8}{|c|}{ INTELIGENCIAS MÚLTIPLES } \\
\hline & & I.NAT & I.MUS & I.MAT & I.INTER & I.CINES & I.LING & I.INTRA & I.VESP \\
\hline MEDIA & 11,8 & 5,9 & 5,8 & 5,6 & 5,8 & 5,5 & 5,2 & 5,6 & 5,5 \\
\hline DESVIACION TÍPICA & 0,8 & 1,6 & 2,1 & 1,7 & 1,7 & 2,0 & 1,9 & 1,8 & 2,0 \\
\hline MODA & 11 & 6 & 4 & 6 & 5 & 6 & 4 & 6 & 4 \\
\hline
\end{tabular}

Asimismo, en la Tabla 2 pueden apreciarse los estadísticos analizados en la variable motivación, creatividad y test de Bender.

\begin{tabular}{|l|c|c|c|}
\hline \multicolumn{4}{|c|}{ Tabla 2 } \\
\hline Motivación, Creatividad y Bender \\
\hline MEDIA & MOTIVACIÓN & CREATIVIDAD & BENDER \\
\hline DESVIACION TÍPICA & 19 & 16,5 & 6,9 \\
\hline MODA & 116 & 4,1 & 3,2 \\
\hline
\end{tabular}


En cuanto, el análisis correlacional realizado entre las variables, se ha utilizado el coeficiente de correlación paramétrico de Pearson, (más de 30 casos) y los datos de las variables obtenidas en la fase pretest. En primer lugar, puede apreciarse en la Tabla 3, no existe relación estadísticamente significativa entre las variables creatividad y Bender en nuestra muestra.

\begin{tabular}{|c|c|c|c|}
\hline \multicolumn{4}{|c|}{ Tabla 3} \\
\hline \multicolumn{1}{|c|}{ Correlación entre Creatividad y Bender } \\
\hline & $\begin{array}{c}\text { TOTAL } \\
\text { CREATIVIDAD }\end{array}$ & $\begin{array}{c}\text { TOTAL } \\
\text { BENDER }\end{array}$ \\
\hline \multirow{2}{*}{$\begin{array}{c}\text { TOTAL } \\
\text { CREATIVIDAD }\end{array}$} & Correlación de Pearson & 1 &,- 010 \\
\cline { 2 - 4 } & Sig. (bilateral) & &, 950 \\
\cline { 2 - 4 } & $\mathrm{N}$ & 40 & 40 \\
\hline
\end{tabular}

En cuanto a la relación entre creatividad y motivación (Tabla 4), puede observarse que se ha encontrado una relación estadísticamente significativa entre las dos variables medidas, siendo esta de tipo positivo.

\begin{tabular}{|c|c|c|c|}
\hline \multicolumn{4}{|c|}{ Tabla 4} \\
Correlaciones entre creatividad y motivación \\
\hline \multicolumn{2}{|c|}{} & $\begin{array}{c}\text { TOTAL } \\
\text { CREATIVIDAD }\end{array}$ & $\begin{array}{c}\text { TOTAL } \\
\text { MOTIVACIÓN }\end{array}$ \\
\hline $\begin{array}{c}\text { TOTAL } \\
\text { CREATIVIDAD }\end{array}$ & Correlación de Pearson & 1 &, $317^{*}$ \\
\cline { 2 - 4 } & Sig. (bilateral) & &, 046 \\
\cline { 2 - 4 } & $\mathrm{N}$ & 40 & 40 \\
\hline
\end{tabular}

En la Gráfica 1 puede verificarse con nitidez dicha relación positiva entre ambas variables.

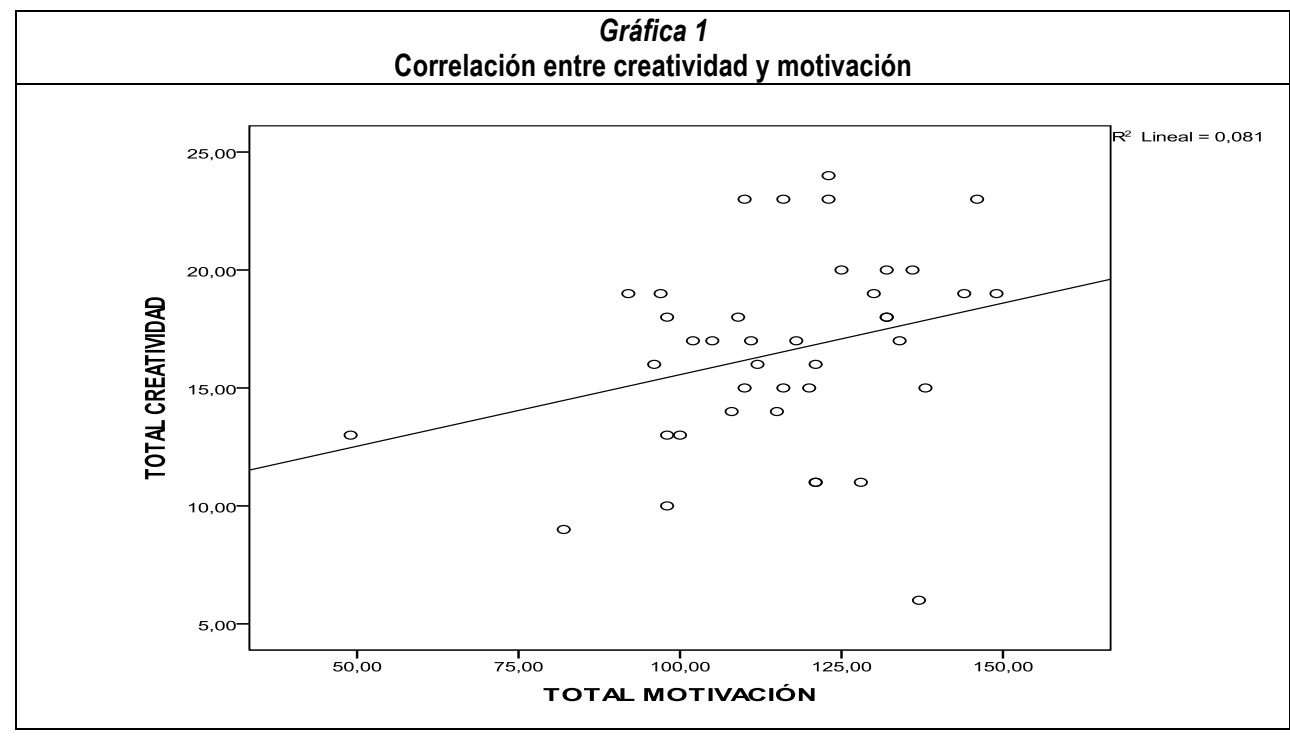

A continuación, se muestran las correlaciones analizadas entre las variables de inteligencias múltiples con el Test de Bender (Tabla 5), no se evidencia relación estadísticamente significativa. 


\begin{tabular}{|c|c|c|c|c|c|c|c|c|c|c|}
\hline & & Correlaciones & ntre $B e$ & $\begin{array}{l}\text { abla } 5 \\
\text { der e ir }\end{array}$ & teligenc & as múlt & & & & \\
\hline & & TOTAL BENDER & I.NAT & I.MUS & I.MAT & I.INTE & I.CIN & I.LING & \begin{tabular}{|l|l|} 
I.INTR \\
\end{tabular} & I.VESP \\
\hline $\begin{array}{l}\text { TOTAL } \\
\text { BENDER }\end{array}$ & $\begin{array}{l}\text { Correlación de } \\
\text { Pearson }\end{array}$ & 1 & ,032 &,- 001 & \begin{tabular}{|l|}
,- 140 \\
\end{tabular} & ,014 &,- 022 & ,032 & ,030 &,- 018 \\
\hline & Sig. (bilateral) & & 847 & ,996 & ,388 & ,933 & ,892 & ,847 & ,856 & ,913 \\
\hline & $\mathrm{N}$ & 40 & 40 & 40 & 40 & 40 & 40 & 40 & 40 & 40 \\
\hline
\end{tabular}

\section{Resultados comparativos}

Para realizar los análisis comparativos pre y post-test se ha empleado prueba $\mathrm{T}$ de Student para grupos relacionados, ya que los dos grupos a comparar están formados por los mismos sujetos, medidos en dos ocasiones distintas (pretest y postest). Además, es necesario contar con un tamaño muestral suficiente (más de 30 casos) y variables dependientes cuantitativas. La tabla 6, muestra las medias y desviaciones típicas antes y después del tratamiento de cada variable dependiente.

\begin{tabular}{|c|c|c|c|c|c|}
\hline \multicolumn{6}{|c|}{$\begin{array}{c}\text { Tabla } 6 \\
\text { Estadísticos de muestras relacionadas }\end{array}$} \\
\hline & & Media & $\mathrm{N}$ & Desviación típ. & Error típ. de la media \\
\hline \multirow[t]{2}{*}{ Par 1} & TOTAL MOTIVACIÓN(pretest) & 115,8500 & 40 & 19,31991 & 3,05475 \\
\hline & TOTAL MOTIVACIÓN (postest) & 119,7000 & 40 & 17,28672 & 2,73327 \\
\hline \multirow[t]{2}{*}{ Par 2} & I.NAT (pretest) & 5,8750 & 40 & 1,57199 & 24855 \\
\hline & I.NAT (postest) & 6,5750 & 40 & 1,44803 & 22895 \\
\hline \multirow[t]{2}{*}{ Par 3} & I.MUS (pretest) & 5,7500 & 40 & 2,14536 & ,33921 \\
\hline & I.MUS (postest) & 6,3000 & 40 & 1,66718 & ,26360 \\
\hline \multirow[t]{2}{*}{ Par 4} & I.MAT (pretest) & 5,6250 & 40 & 1,65928 & ,26235 \\
\hline & I.MAT (postest) & 6,6000 & 40 & 1,25678 & 19871 \\
\hline \multirow[t]{2}{*}{ Par 5} & I.INTER (pretest) & 5,8000 & 40 & 1,69766 & ,26842 \\
\hline & I.INTER (postest) & 6,3250 & 40 & 1,54235 & 24387 \\
\hline \multirow[t]{2}{*}{ Par 6} & I.CINES (pretest) & 5,4500 & 40 & 2,01214 & ,31815 \\
\hline & I.CINES (postest) & 6,2000 & 40 & 1,58842 & ,25115 \\
\hline \multirow[t]{2}{*}{ Par 7} & I.LING (pretest) & 5,1500 & 40 & 1,91552 & ,30287 \\
\hline & I.LING (postest) & 5,9000 & 40 & 1,80881 & ,28600 \\
\hline \multirow[t]{2}{*}{ Par 8} & I.INTRA (pretest) & 5,6000 & 40 & 1,83694 & ,29045 \\
\hline & I.INTRA (postest) & 6,4000 & 40 & 1,48151 & ,23425 \\
\hline \multirow[t]{2}{*}{ Par 9} & I.VESP (pretest) & 5,4750 & 40 & 2,01262 & ,31822 \\
\hline & I.VESP (postest) & 6,5500 & 40 & 1,64784 & ,26055 \\
\hline \multirow[t]{2}{*}{ Par 10} & TOTAL CREATIVIDAD (pretest) & 16,5250 & 40 & 4,11992 & ,65142 \\
\hline & TOTAL CREATIVIDAD (postest) & 19,1750 & 40 & 3,86229 & ,61068 \\
\hline
\end{tabular}

Se evidencia un aumento entre las medias de pretest y postest en todas las variables analizadas. No obstante, para confirmar que esas diferencias sean significativas debe analizarse la información de la Tabla 7, Pruebas de muestras relacionadas (comparación un mismo grupo en dos fases). 


\begin{tabular}{|c|c|c|c|c|}
\hline \multicolumn{5}{|c|}{$\begin{array}{c}\text { Tabla } 7 \\
\text { Correlaciones de muestras relacionadas }\end{array}$} \\
\hline & & $\mathrm{N}$ & Correlación & Sig. \\
\hline Par 1 & TOTAL MOTIVACIÓN pre y posttest & 40 & ,647 &, 000 \\
\hline Par 2 & I.NAT pre y posttest & 40 &, 551 &, 000 \\
\hline Par 3 & I.MUS pre y posttest & 40 & ,731 &, 000 \\
\hline Par 4 & I.MAT pre y posttest & 40 &, 725 &, 000 \\
\hline Par 5 & I.INTER pre y posttest & 40 & ,623 &, 000 \\
\hline Par 6 & I.CINES pre y posttest & 40 &, 581 &, 000 \\
\hline Par 7 & I.LING pre y posttest & 40 & 633 &, 000 \\
\hline Par 8 & I.INTRA pre y posttest & 40 &, 588 &, 000 \\
\hline Par 9 & I.VESP pre y posttest & 40 &, 538 &, 000 \\
\hline Par 10 & TOTAL CREATIVIDAD pre y posttest & 40 & ,674 &, 000 \\
\hline
\end{tabular}

En la Tabla 8 puede apreciarse si las diferencias son estadísticamente significativas en la fase pre y post- test tras la intervención. En este caso, excepto la motivación, existen diferencias significativas en las puntuaciones de pretest y postest y esas diferencias son a favor del postest. Es decir, los estudiantes obtienen puntuaciones más altas en el postest.

\begin{tabular}{|c|c|c|c|c|c|c|c|c|c|}
\hline \multicolumn{10}{|c|}{$\begin{array}{c}\text { Tabla } 8 \\
\text { Prueba de muestras relacionadas }\end{array}$} \\
\hline & & \multicolumn{5}{|c|}{ Diferencias relacionadas } & \multirow[t]{3}{*}{$t$} & \multirow[t]{3}{*}{$\mathrm{Gl}$} & \multirow{3}{*}{$\begin{array}{c}\text { Sig. } \\
\text { (bilateral) }\end{array}$} \\
\hline & & \multirow[t]{2}{*}{ Media } & \multirow[t]{2}{*}{$\begin{array}{l}\text { Desviación } \\
\text { típ. }\end{array}$} & \multirow[t]{2}{*}{$\begin{array}{l}\text { Error típ. de } \\
\text { la media }\end{array}$} & \multicolumn{2}{|c|}{$\begin{array}{c}95 \% \text { Intervalo de } \\
\text { confianza para la } \\
\text { diferencia }\end{array}$} & & & \\
\hline & & & & & Inferior & Superior & & & \\
\hline Par 1 & T. MOTIV pre y post & $-3,8500$ & 15,49781 & 2,45042 & $-8,80644$ & 1,10644 & $-1,571$ & 39 & ,124 \\
\hline Par 2 & I.NAT pre y post &,- 70000 & 1,43581 & ,22702 & $-1,15919$ &,- 24081 & $-3,083$ & 39 &, 004 \\
\hline Par 3 & I.MUS pre y post &,- 55000 & 1,46672 & ,23191 & $-1,01908$ &,- 08092 & $-2,372$ & 39 &, 023 \\
\hline Par 4 & I.MAT pre y post &,- 97500 & 1,14326 & , 18077 & $-1,34063$ &,- 60937 & $-5,394$ & 39 &, 000 \\
\hline Par 5 & I.INTER pre y post &,- 52500 & 1,41399 & ,22357 &,- 97721 &,- 07279 & $-2,348$ & 39 & ,024 \\
\hline Par 6 & I.CINES pre y post &,- 75000 & 1,69085 &, 26735 & $-1,29076$ &,- 20924 & $-2,805$ & 39 &, 008 \\
\hline Par 7 & I.LING pre y post &,- 75000 & 1,59727 & ,25255 & $-1,26083$ &,- 23917 & $-2,970$ & 39 &, 005 \\
\hline Par 8 & I.INTRA pre y post &,- 80000 & 1,53923 & ,24337 & $-1,29227$ &,- 30773 & $-3,287$ & 39 & ,002 \\
\hline Par 9 & I.VESP pre y post & $-1,0750$ & 1,78868 & ,28281 & $-1,64705$ &,- 50295 & $-3,801$ & 39 &, 000 \\
\hline Par 10 & T.CREA pre y post & $-2,6500$ & 3,23086 &, 51084 & $-3,68328$ & $-1,61672$ & $-5,187$ & 39 &, 000 \\
\hline
\end{tabular}

\section{Discusión}

Los resultados de la tabla uno, muestran que el desarrollo de las inteligencias múltiples es similar en promedio y en desviación. Esto permite suponer que actividades propuestas en el aula debe cambiar, pues el método tradicional no permite desarrollar las inteligencias en un alto nivel. Esto coincide con el artículo realizado por Seligman (2013). En la tabla uno y la seis se observó en los datos pretest que la inteligencia con mejor promedio fue la inteligencia naturalista y la de menor promedio fue la inteligencia lingüística, esto verifica que hay unos dominios cognitivos sobre otros (fortaleza y debilidades), así vemos confirmada la propuesta de Gardner (1999) cuando estableció diferentes perfiles de inteligencia para cada sujeto.

Los resultados de la tabla dos en la variable creatividad, muestran una media por encima de 12 , eso indica que el $53 \%$ de los estudiantes pertenecientes a la muestra tiene potencial creativo alto. En esta misma tabla se hallan los resultados de la prueba de Bender, muestran una desviación grande, esto se interpretar como un grupo heterogéneo, es decir, hay estudiantes con buena percepción visomotriz y otros que por el contrario 
tiene grandes dificultades. Finalmente está el resultado de la motivación donde la media indica que la mayoría de estudiantes están motivados.

Respecto a los análisis correlacionales, se encontró relación estadísticamente significativa entre las variables creatividad y motivación, concretamente de 0,317 ( $p=0,46<0,05)$. Esta relación es positiva y baja, lo cual indica que cuando hay mayor motivación hay más creatividad y viceversa. Al igual que puntuaciones bajas en motivación, están relacionadas con bajas puntuaciones en creatividad y viceversa. Estos hallazgos se hallan en consonancia con las conclusiones de Acosta (1998) quien afirma que ambas variables se hallan relacionadas entre sí, y que además se relacionan con el rendimiento académico. Thome (2008) va más allá en esta relación, ya que argumenta que ambas variables se relacionan y son indispensables para ayudar a nuestros alumnos a que sean capaces de pensar por sí mismos o de afrontar pensamientos complejos necesarios para la vida.

En cuanto a los datos comparativos, como se ha visto en el apartado de resultados, el uso del Tangram como recurso didáctico ha tenido beneficiosos en la creatividad y las IM en la muestra estudiada. Estos resultados positivos están en la línea de Martínez e Irene (2013) que recientemente mostraron la eficacia de una metodología activa para el aprendizaje de las matemáticas. Según estos autores, una metodología participativa y práctica, usando entre otros, el recurso del Tangram, ayudará al docente a comunicarse eficazmente con los alumnos y éstos a la vez serán capaces de asimilar el conocimiento necesario y reflejarlo en el desarrollo de sus habilidades. La inteligencia múltiple que más incremento su puntuación, fue la viso-espacial. Este dato es coherente con el argumento de LalienaTolosana (2013) quien afirma que dentro de los recursos de entretenimiento más importantes relacionados con la habilidad viso-espacial, desarrollo la lógica, avance de las IM y los procesos de enseñanza-aprendizaje de la geometría, se encuentra el Tangram.

También el uso del Tangram ha mejorado la puntuación en creatividad de los alumnos de la muestra. Así pues, se ha pasado de una puntuación de 16.52 a 19.57. Estos hallazgos se verifican con las afirmaciones de Benítez (2008) que afirma que el tangram constituye un auténtico recurso para dar rienda suelta a la creatividad.

El uso del Tangram no ha logrado los efectos esperados en la variable motivación. A pesar de que se ha pasado de 115 a 119 puntos, los resultados no son estadísticamente significativos, ya que la diferencia en la comparación tienen demasiado margen de error $(p=.124)$. Estos hallazgos no apoyan las aportaciones de Bravo (2009) quien sugiere una relación positiva entre el uso del Tangram y la motivación. Quizás, este resultado se deba a que la motivación medida en la presente investigación infiera procesos atribucionales que requieren de un mayor tiempo para que se muestren los cambios.

\section{Conclusiones}

El objetivo general de esta investigación fue comprobar si la aplicación de un programa basado en diferentes actividades del Tangram potencia y desarrolla las IM, la creatividad y la motivación de los alumnos que conforman la muestra. Los resultados analizados, demuestran que sí, excepto en la variable de motivación.

Para llegar a cumplir el objetivo general, se consiguió conocer el nivel de los alumnos de la muestra en cada una de las IM, analizar su grado de motivación, describir el nivel de 
creatividad y establecer el nivel de percepción viso-motriz en el que se encuentran y así crear un programa de intervención basado en la manipulación del Tangram para enseñar conceptos matemáticos.

Esta investigación ha estudiado la posible relación entre las variables del desarrollo visomotriz de los estudiantes con las inteligencias múltiples y la creatividad y no ha encontrado una relación estadísticamente significativa en ninguno de los casos.

También se ha analizado si existe relación entre la creatividad y la motivación en la muestra estudiada y se ha encontrado una relación estadísticamente significativa entre ambas variables de tipo positivo.

Por todo ello y teniendo en cuenta las limitaciones que presenta este estudio, sobre todo en cuanto al número de la muestra, se puede afirmar que el uso de Tangram puede ser una herramienta de gran utilidad para la enseñanza de las matemáticas. Sería interesante y provechoso continuar estudiando la efectividad de este tipo de herramientas en diferentes contextos.

\section{Referencias}

Acosta, M. (1998). Creatividad, motivación y rendimiento académico. Málaga: Ediciones Aljibe.

Bender, L. (1977). El Test Guestáltico Visomotor: Usos y Aplicaciones Clínicas. México: Paidós.

Benítez, N. (2008). Once estrategias para divertirse y aprender con Triángulos equiláteros. Encuentro Colombiano de Matemática Educativa Valledupar, Colombia.

Bravo, C. (2009). Los Juegos Como Estrategia Metodológica en la Enseñanza de la Geometría, en Estudiantes de Séptimo Grado de Educación Básica. Estado Nueva Esparta: Universidad de oriente.

Cabrera Astudillo, M. A., Lluilema, N., \& Carmen, M. (2011). Los Juegos Educativos con Materiales Concretos para el Aprendizaje de las Matemáticas en los Estudiantes de Cuarto Año de Educación Básica de la Escuela "Medalla Milagrosa" de la Provincia de Chimborazo, Cantón Guano, Parroquia la Matriz durante el Año 2010-2011.

Cruz, M.S., Garaigordobil, M. \& Maganto, C. (2001). Análisis evolutivo de la coordinación visomotora y sus relaciones con inteligencia, estilo cognitivo y atención. Revista Española de Orientación y Psicopedagogía, 12(21), 73-88.

Gardner, H. (1983). Estructura de la mente: la teoría de las múltiples inteligencias. México: Fondo de cultura de España.

Gardner, H. (1995). Mentes creativas: una anatomía de la creatividad vista a través de las vidas de S. Freud, A.Einstein, P. Picasso, I. Stravinsky, T.S. Elliot, M. Graham, M. Gandhi. Barcelona: Ediciones Paidós Ibèrica, S.A

Gardner, H. (1999). Mentes extraordinarias. Cuatro retratos para descubrir nuestra propia excepcionalidad. Barcelona: Editorial Kairós,

García, F. \& Doménech, F. (2012). Motivación, aprendizaje y rendimiento escolar. Revista electrónica de motivación y emoción, 1(0). 
Jaramillo, J.A. \& Ruíz, J. A. (2012). Una estrategia pedagógica para motivar procesos de aprendizaje en estadística descriptiva de básica secundaria. Revista de investigación Silogismo, 1(10),.

Laliena Tolosana, F. J. (2013). Dificultades en el proceso de la enseñanza-aprendizaje de la geometría en $1^{\circ}$ de la ESO. Tesis de maestría, Universidad Internacional de la Rioja.

Linares, A. (2013). ¿Por qué somos tan malos en matemáticas? El Tiempo sección de educación.

Manassero, M. A. \& Vázquez Alonso, A. (1996). Validación de una escala de motivación basada en la teoría atribucional de Weiner. Psicothema, 10(2), 217-235.

Jaramillo, M. I. (2013). La didáctica dinámica de la matemática incide en el aprendizaje cognitivo de los estudiantes de octavo grado de educación básica de la unidad educativa "Ingapirca" de la parroquia Santa Rosa de Cuzubamba del Cantón Cayambe, provincia de Pichincha. Tesis de Licenciada, Universidad Técnica de Ambato.

Montilla, P. (1997). Creatividad humana enfoques y bases neurobiológicas. Revista de la cátedra de psicología médica y psiquiatría y U D de historia de la medicina, 8(1-2), 13-32.

Ortega, A. (2012). Creatividad y habilidad comunicativa. México: Editorial De la Salle.

Pekrun, R. (1992). The impact of emotions on learning and achievement: Towards theory of cognitive/motivational mediators. Applied psychology: An international review, 41(4), 359376.

Pradas, J. y Pardo, F. (2013). Para qué una reforma educativa: Una consideración intempestiva de la desde la Pedagogía crítica.

Seligman, M. (2013). Inteligencias múltiples en el aula.

Weiner, B. (1974). Achievement motivacion and attribution. Morristown, N.J: General Learning Press. 